\title{
Considerations Regarding Dental Implant Site Preparation using Osseo densification Osteotomy. Critical Review
}

\author{
Adel A Al Asfour¹, Moosa Abuzayeda2*, Colin Alexander Murray ${ }^{3}$, Mohammad Kamal ${ }^{4}$ and Gregor-Georg \\ Zafiropoulos ${ }^{5}$
}

${ }^{1}$ Associate Professor, Department of Surgical Sciences, Faculty of Dentistry, Kuwait University, Kuwait

${ }^{2}$ Associate Professor, Department of Prosthodontics, College of Dental Medicine, MBR University, UAE

${ }^{3}$ Professor, Department of Preventive and Restorative Dentistry, University of Sharjah, UAE

${ }^{4}$ Assistant Professor, Department of Surgical Sciences, Faculty of Dentistry, Kuwait University, Kuwait

${ }^{5}$ Professor, Department of Surgical Sciences, Faculty of Dentistry, Kuwait University, Kuwait

Submission: June 25, 2020; Published: July 10, 2020

*Corresponding author: Moosa Abuzayda, Associate Professor, Department of Prosthodontics, College of Dental Medicine, Mohammed Bin Rashid University, Dubai HealthCare City, Dubai, UAE

\section{Abstract}

Conventional osteotomies for implant placement are typically prepared with implant drills originally designed for non-dental procedures. Such drill designs have proven efficacy for implant placement, resulting in good implant success rates over many years. Conventional drill designs used in oral implantology remove bone to create space for implant insertion. While these osteotomy drills effectively remove bone, they may not always create circumferential osteotomy sites. Therefore, implant insertion torques may be lower than ideal, possibly compromising primary implant stability (PIS). This in turn may adversely affect implant osseointegration (OI). Osteotomies performed in areas with narrow bone anatomy may also cause a dehiscence, which potentially further reduces PIS. Furthermore, additional bone grafting procedures may be required thereby prolonging healing time before implant restorations can be completed. More recently, a novel biomechanical bone preparation technique called "osseodensification" (OD) has been proposed. Unlike traditional dental implant drilling procedures, osseodensification does not remove bone tissue. Conversely, bone tissue is retained and compacted in outwardly expanding directions within the osteotomy preparation site. This is similar to the effect of a traditional hammer osteotome but with less adverse effects. When the OD bur is rotated at high speed in a counterclockwise direction, a dense bone tissue layer is created along the walls and base of the osteotomy site. The formation of this dense compacted bone layer may contribute towards faster OI by enhancing PIS. This review discusses the potential advantages of OD and critically evaluates the currently available literature investigating this interesting implant bed preparation technique.

Keywords: Osseodensification; Dental implants; Implant stability; Osseointegration

Abbreviations: PIS: Implant Stability; OI: Osseointegration; BIC: Bone-to-Implant Contact; OD: Osseodensification; SD: Standard Surgical Drilling osteotomy; SO: Summers' Osteotomy; CCW: Counterclockwise; CW: Clockwise rotation; ISQ: Implant Stability Quotients; CTG: Connective Tissue Graft; CM: Collagen-based Matrice; STTh: Soft-Tissue Thickness' PD: Piezoelectric Device; OT: Osteotome

\section{Introduction}

With the popularization of implant-supported restorations, clinicians seeking to perform them are increasingly being confronted with challenges that impede implant placement. Such challenges include alveolar ridge resorption following tooth extraction, bone defects, and atrophic areas. A variety of techniques have been developed to enable implant rehabilitation in cases complicated by these challenges, such as guided bone regeneration with barrier membranes, particulate autografts, allografts, alloplastic restoration, and xenografts [1-3]. Endosteal implants are anchored into bone tissue. Their long-term success and retention depend firstly on primary implant stability (PIS) and, ultimately, on the biological stability mediated by osseointegration (OI) [4-9]. PIS refers to the avoidance of micromovements at the implant-bone interface during early postoperative bone remodeling, which is determined by physical interactions of the implant with surrounding bone tissue [10]. It degrades gradually after the initial implant placement [4-9]. Meanwhile, a transition occurs wherein bone remodeling during OI - defined as the development of a mechanical connection between living bone tissue and an implant body - brings the implant surfaces into tight apposition with stabilizing bone tissue [4-9]. 
Clinical OI research, translated from preliminary studies in animal models, has demonstrated that reduced cortical bone shell adheres to an implant directly (without interceding tissue) during postoperative immobilization [11-14]. Long-term functional OI depends upon the functional and biological characteristics of both the implant and implantation site [4-9]. That is, OI is dependent upon the implant material and geometry as well as on the condition and density of the alveolar bone surrounding the implant $[5,8,15]$. Principally, successful OI requires adequate microscopic bone-toimplant contact (BIC) and adequate bone quality at the implant interface to maintain BIC. Low bone density or poor surgical technique can result in unsustainable BIC [6-8,11,12]. Indeed, OI has been shown to correlate strongly with bone density and to improve with the use of greater peak insertion torque [12,15-17]. Meanwhile, maintenance of ample collagen and minerals in the implant site may accelerate healing while supporting PIS until OI has been completed [4].

Conversely, a poor osteotomy site preparation can result in OI-impeding bone necrosis [11-14]. Given the aforementioned dependencies, thus, implantologists are keen to optimize the implant site for bone retention while preserving microscopic bone structure in osteotomy preparations $[14,18]$. Accordingly, an endosteal implant site preparation approach known as osseodensification (OD) drilling has been introduced with the goal of facilitating subsequent OI $[15,19]$. The aim of OD is to create an environment that enhances PIS through densification of osteotomy site walls by non-subtractive drilling, resulting in condensed autogenous bone [16]. The rationale for the OD osteotomy is that densification of bone tissue that will be in direct contact with an endosteal device immediately upon its insertion will facilitate device-bone interlocking via enhanced BIC and the promotion of rapid new bone growth owing to osteoblasts nucleating on disrupted bone in close proximity to the implant [20]. The aim of the present review was to compare and to critically discuss the outcomes of implant sites prepared using OD to those prepared with standard osteotomy drilling. In addition, given the limited nature of the literature related to OD primarily derived from clinical case reports, in vitro and animal studies, we provide a summative discussion of the currently available data.

\section{Osteotomy preparation techniques}

Until recently, there have been 2 main osteotomy preparation techniques: the standard surgical drilling (SD) osteotomy, which involves bone tissue extraction, and Summers' non-extraction osteotomy (SO) [21-23]. SD is performed with conventional drills adapted for dental use from metal- and woodworking. SO avoids bone extraction, but risks fracture, bone displacement, and surgical trauma. Given these limitations, a new osteotomy preparation (osseodensification, OD) was developed in which specially designed burs are used to compact, rather than excavate, bone and to autograft the particulate bone generated, yielding a bone-preserving osteotomy [15]. For SD osteotomy preparation, a space is created for the implant by drilling and excavating bone tissue with commercially available instruments modified for dental use [16]. The drill consists of a diameter shank with a chiselededge tip. The SD drill is fitted with outward projecting cutting lips and spiral guides (lands) and flutes (channels) that extract debris from the created space. Attached to the flutes is a positively angled rake that removes material with every flute rotation [16]. The coincident excavation of healthy bone reduces PIS, compromises geometric accuracy, and prolongs healing. If sufficient irrigation and cooling are not provided, SD can also generate excess heat. Thus, although SD is the most common osteotomy preparation method, clinicians are interested in alternative methods that do not sacrifice extant healthy bone tissue.

The SO involves bone-augmented osteotome (OT) sinus-floor elevation wherein vertical bone height is gained by relocating excavated bone into the osteotomy site prior to sinus elevation [20]. In this technique, osteotomes of increasing diameter are introduced sequentially to expand the implant site, and particulate graft material is added prior to advancing each OT [20]. The SO approach avoids bone extraction, but carries a risk of fracture, bone displacement, and surgical trauma [20]. An 8\% implant failure rate has been reported for $\mathrm{SO}$, with failures being attributed to bone-implant friction and osteotomy site fractures [24]. In osseodensifection (OD), a newly developed bone preparation method, the radial land acts on a noncutting edge and expands the osteotomy while increasing bone density. OD preserves bone bulk, facilitates compaction autografting, and deforms trabecular bone in an outward strain, which result in alveolar ridge plastic expansion. OD burs are grooved to limit heating during bone drilling. OD burs have reduced-size shanks in order to improve the acuity of osteotomy entry [16,19].

In contrast to the conventional drilling, the OD process creates an osteotomy using tapered, multi-grooved burs which present cutting chisels and tapered shanks allowing them to progressively increase the diameter of the osteotomy site by drilling deeper into the bone, which controls the expansion process. For the OD technique, different burs are utilized at a negative rake angle to create a layer of dense bone surrounding the area of the osteotomy [16,19]. Densification takes place at high speed (8001,200 rotations per minute) with counterclockwise (CCW) bur rotation. It has been shown that CCW rotation is more efficient for the densification process than clockwise rotation (CW) and thus is suggested for low density bones [9]. According to preliminary results, OD enhances both BIC and PIS, enabling faster healing and higher OI success rates, because the approach achieves an insertion torque $(45 \mathrm{Ncm})$ and 68 implant stability quotients (ISQ) $[9,25,26]$. It has been reported that OD preserves bone bulk and could reduce the waiting time in loading and also helps ridge expansion while maintaining alveolar ridge integrity, thereby allowing implant placement in autogenous bone and achieving adequate PIS [15,16,19,25]. 


\section{Bone density and implant bed preparation}

Implant success is dependent on both the biologic tissue response and associated mechanical factors [5,8,11,12,27-30]. PIS is a major requisite for both direct bone deposition onto the surface of the implant and its integration with mineralized tissue $[4,5,14,31,32]$. PIS lowers the level of implant micromotion, which, in turn, allows undisturbed healing and OI [28]. Studies have demonstrated that PIS and implant survival rates are determined by the bone density, the surgical technique applied, and the implant design [16,26,27,29,33-36]. Although PIS and OI can be predictably achieved in dense bone, it is often challenging to achieve the same in areas with low-density bone. PIS can decrease remarkably in low-density bone, jeopardizing the OI process and risking failure [37]. Achieving PIS in areas with low-density bone is often challenging to the clinician. Previous research has suggested that modified surgical protocols might be beneficial in such situations. Low-density bone implant sites have been reported as the greatest potential risk factor for implant loss when working with standard drilling (SD) protocols. Therefore, depending on the bone density at the scheduled implant position, drilling procedure which undersizes the implant bed preparation could be chosen to optimize PIS [35,38]. Others have proposed bone condensation using osteotomes and using a final drill size smaller than recommended [21-23,37]. However, the insertion of implants using osteotomes can result in the occurrence of microfractures in the peri-implant bone compared with implants inserted using a conventional drilling method [21-23].

In a recent study, Turkyilmaz and McGlumphy found significant correlations between bone density and PIS [27]. This agrees with a previous study by Turkyilmaz et al. [39] and indicated that clinicians may predict PIS and modify their treatment plans before implant placement, where the bone density is low. Alghamdi et al. [35] evaluate the survival rate of implants placed using undersized implant site preparation in areas with low-density bone. For this purpose, 26 implants were placed according to a standard drilling protocol as control group in comparison to 26 implants placed in low-density bone using an undersized implant size preparation. The results of this study suggest that placement of implants by an undersized placement in sites with low-density bone is beneficial in enhancing PIS and improving the implant survival rate. Other studies have also investigated the bone healing pattern and OI of implants inserted with an undersized implant size preparation and in comparison, to implants placed with the conventional procedure $[35,37,38,40]$. The overall results showed that a significantly higher BIC was achieved in implants installed using an undersizing approach. This increased bone contact was suggested to be due to the occurrence of small bone fragments, which are created during the undersized installation of an implant in the alveolar bone bed. These bone fragments were confirmed to possess osteogenic potential and could probably be considered as a kind of autograft $[27,40,41]$.
In a study of 72 osteotomies in a porcine tibia model, was tested whether OD can yield better PIS, BIC and bone mineral density than SD or SO. They confirmed that insertion and removal torque were indeed greater with OD than with SD or So. Furthermore, biomechanical stability assessments using histomorphology, and microcomputed tomographic imaging demonstrated that OD resulted in significantly better treatment outcomes, including healing, than the other 2 techniques [16]. Applying Donath and Rohrer's cutting/grinding method was observed very little trabecular bone around the implant after SD, some compression where SO had been applied, and complete compression with a regular uniform pattern after OD. The study showed that OD enhanced BIC by more than twofold compared to the other methods: $60.3 \%, 40.7 \%$, and $16.3 \%$ (OD, SO, SD respectively) $[13,42]$. The bone volume percentage of the 2 -mm margin surrounding the implant body was also greater for OD than for SO or SD. Lahens et al. [26] employed an in vivo sheep iliac crest model (72 implants, half with 12 weeks healing, and half with 3 weeks healing) to evaluate OD technology with respect to time and implant area handling in low-density bone. Fixtures prepared with OD exhibited greater PIS than those prepared with SD. The OD procedures were successful without any complications. The authors concluded that OD yields very good PIS, especially when applied in the CCW direction, owing to the spring-back effect caused by bone elasticity [26]. In another sheep model study, Trisi et al. [33] examined whether CCW OD yields improved bone density around the implant site relative to SD and investigated OD effects on ridge width and implant success two months after surgery. A 30\% higher bone volume percentage was observed for the OD group compared to SD group.

Subsequently, in an examination of biomechanical OD approaches in areas with low bone mass, Lahens et al. [9] studied the relationship of OD with PIS and successful OI in conical and parallel-walled implants in five sheep, with each animal receiving multiple SD, CW OD, and CCW OD implants. They evaluated bone tissue occupancy and tissue-implant bonding. They found that insertion torques were higher $(\sim 100 \mathrm{Ncm})$ for the OD group than for the SD group ( $\sim 25 \mathrm{Ncm})$, irrespective of implant geometry. Moreover, they found that OD resulted in good PIS and tissue-implant bonding quality owing to the spring-back effect produced by instrumentation autografting. Histological analysis showed that both SD and OD led to autograft particles around the implant, with the implants placed after CCW OD exhibiting more autograft particles than those placed after CW OD or SD. OD had a greater insertion force than SD, and BIC was greater for both OD techniques (CCW-OD 70\% BIC and CW-OD 60\%) than for SD (50\%). Bone-area-fraction occupancy was independent of drilling method, but higher for parallel implants than for conical implants. Six weeks postoperatively, new bone formation and remodeling were observed for all groups.

A year later (2017), Lopez et al. [43] performed a study to evaluate whether CCW OD can enhance spinal surgical hardware 
fixation relative to SD. They used biomechanical evaluation of pull-out strength and histomorphometry of bone mass and quality to examine whether improvements in hardware fixation can improve surgical outcomes in 12 65-kg sheep. Early low occupancy values in the first 3 weeks of healing after SD were attributed to bone excavation during drilling. Bone growth was observed after 3 weeks at SD sites. Notwithstanding, at week 6, OD yielded significantly higher bone tissue occupancy than SD. They established that pullout strength and bone tissue occupancy were greater with OD than with SD. This particular study was unusual in that it tested spinal surgical hardware; The OD bur significantly improved the surgical therapy. These results proved that OD should be considered for spinal surgical instrumentation. With a focus on macrogeometry and surgical therapy, Alifarag et al. [24] examined whether OD can hasten the establishment of satisfactory PIS. They found greater insertion forces, BIC, and bone-area-fraction occupancy for both CW and CCW OD relative to those observed with SD. The osteotomies prepared with OD exhibited greater PIS than those prepared with OD.

Oliveira et al. [44] investigated the impact of OD instruments on the external implant-site walls in contact with the implant and the implant surfaces themselves. SD, CW-OD, and CCW-OD were performed in sheep (all three approaches in 6 sheep, withinsubject comparisons). The CCW-OD osteotomy had the highest insertion force value, followed by CW-OD, and SD was associated with the lowest force value, irrespective of the implant surface. Although BIC was consistent over time, the percentage of bone tissue occupancy increased in the sixth week. Relative to the SD sites, they observed better-quality implant-tissue bonding and OI with OD than with SD [44]. Tian et al. [45] compared osteotomy preparation effectiveness in OD-prepared versus SD-prepared implant sites in horizontally atrophic mandibular ridges following premolar extraction in porcine models. The obtained results showed a significantly greater BIC in the OD group $(62.5 \%)$ compared to the traditional osteotomy preparations $(31.4 \%$; $\mathrm{p}=0.018$ ). The authors described OD-associated advantages with respect to PIS and OI, as evidenced by biomechanical and histologic analyses, respectively.

\section{Clinical Observations and Reviews}

In 2015, Huwais [15] reported the achievement of successful OI after 14 weeks of healing in the case of a 62-year-old male patient treated with OD. Two implants were installed to replace 2 missing teeth. The patient consented to osteotomy with ridge expansion employing OD with immediate implant placement and possible later buccal bone grafting. Furthermore, it was observed that ridge expansion had been facilitated by OD. The achievement of rehabilitation with OD in only 14 weeks is remarkable given that the typical treatment plan for such a patient requires $30 \sim 50$ weeks (i.e., edge growth for 6 9 months with square uniting or guided bone recovery, followed by implant placement, healing, and finally tooth restoration). PIS and successful OI were retained 1 year later and observations made at a 2-year followup examination confirmed a stable treatment outcome. In a case study, was described the successful use of CCW-OD in a senior male with low implant-site bone density. Notably, good bone preservation and PIS were achieved. The recommended insertion torque value of $45 \mathrm{Ncm}$ and ISQ of 68 were achieved with the OD approach, enabling early implant loading to be completed [46].

Koutouzis et al. [47] evaluated bone expansion following placement of 28 implants through OD osteotomy in 21 patients. The alveolar ridge width was measured at the level of the crest and $10 \mathrm{~mm}$ apical to the crest before and after OD. Expansion values were grouped into the following three (3) groups according to the initial alveolar ridge width: group 1: 3 - $4 \mathrm{~mm}$, group 2: 5 - 6 $\mathrm{mm}$, and group 3: 7 - $8 \mathrm{~mm}$. A significant difference in the mean expansion value at the coronal aspect of the ridge between group 1 , group 2, and group 3 was found $(2.83 \mathrm{~mm}, 1.5 \mathrm{~mm}$, and 1.14 $\mathrm{mm}$ respectively; $\mathrm{p}<0.05$ ). It was concluded that $\mathrm{OD}$ can allow ridge expansion and that greater expansion can be expected at the crest in narrow ridges with adequate trabecular bone volume. De Mello Machado et al. [48] evaluated radiographs performed preoperatively, trans-operatively, and immediately after implant installation in a case study of a 44-year-old man who underwent tooth extraction, CCW-OD, and immediate tooth implantation at tooth site \#26 due to root fracture. Observations in axial images revealed that OD resulted in PIS roughly threefold that obtained with SD and markedly better BIC percentage than that obtained with SD. The authors expressed optimism regarding OD enabling immediate implant loading owing to the good PIS it can yield. Da Rosa et al. [49] evaluated the combined use of the immediate dentoalveolar restoration technique and the implant placement using OD to improve PIS at sites with severe alveolar bone loss in two clinical cases. The findings were analyzed by clinical assessment, photography, radiography, and computed tomography scans and led to the conclusion that the use of OD could improve PIS, as measured by higher insertion torque.

In an animal, i.e. sheep, model Witek et al. [50] evaluated the effect of osteotomy preparation by conventional versus OD instrumentation on osteotomy healing. Fifteen osteotomies were prepared in the left ilium of the sheep and three different drilling techniques were utilized: (1) conventional/regular drilling (as recommended by manufacturer); (2) OD-CW; and (3) OD ODCCW. Drillings were performed at 1,100 rpm with saline irrigation. Qualitative histomorphometric analysis of the osteotomies after six weeks did not show any healing impairment due to the instrumentation. Histologic analysis showed bone remodeling and growth in all samples, irrespective of osteotomy preparation technique, with the presence of bone chips observed along the length of the osteotomy wall in sites subjected to OD drilling. Almutairi et al. [51] investigated in vitro the effect of OD and different thread designs on PIS. Implants were divided into four groups and each group was made of 12 implants with a different thread design: V-shaped, trapezoid, buttress, and reverse buttress. 
The implants were inserted in 4-mm thick cancellous bone slices obtained from the head of cow femur bone. The osteotomies were prepared by SD and by OD drilling. Each inserted implant was then tested for primary stability using the Periotest.

The results of this study revealed no statistically significant difference between the Periotest readings for the implants in each category placed in either the OD or the regular SD osteotomies. However, it was found that implants placed in SD osteotomies had a significantly better primary stability than the implants placed in OD ones. It was concluded that OD is not necessary in situations where there is bone of good quality and quantity. In a systematic review of 195 reports, Pai et al. [25] determined that, relative to SD, OD resulted in smaller-sized osteotomies than SD together with greater bone density, bone volume percentages, and BIC, characteristics that enhance implant stability. Greater bone density led to increased insertion torque, resulting in reduced micromotion and therefore improved PIS. The studies summarized by Pai et al. [25] concluded that OD creates a broadened osteotomy that conserves and compacts bone mass, which maintains PIS thereby leading to successful OI. In a recent systematic review or 29 studies, Tretto et al. [52] evaluated the influence of instruments used for implant site preparation on the bone-implant interface, namely conventional drills (CDs) used for SD, osteotomes, piezoelectric devices (PDs), and Er: YAG laser, and OD burs. Meta-analyses BIC revealed no difference in BIC, crestal bone as well as in implant survival between osteotomies generated with CDs versus those generated with the other techniques.

El-Kholey \& Elkomy [53] investigated the possible association between the drilling technique and proper implant integration and survival in areas with low bone density. Results of fifteen studies were analyzed - seven in vitro and eight clinical - performed with undersized, osteotomes, piezosurgery, and OD drilling, as these four techniques were found in the literature to enhance implant OI in low-density bone. Owing to the methodological variation, meta-analysis was not performed. The obtained results showed that the four drilling protocols were effective in increasing PIS, but the long-term outcome is comparable with that of the conventional SD. The investigators concluded that there was a weak evidence suggesting that any of the previously mentioned surgical techniques could enhance successful OI and survival of the implants placed in low-density bone. Furthermore, Elsayyad \& Osman [54] in their 2019 review, attempted to make a critical appraisal of the studies relating to OD in the literature. However, they reported that studies advocating OD were sparse, and mainly animal studies of low-evidence level, with high risk of bias and low correlation between animal models and human bone. The authors concluded that although OD seems to be a promising technique, the findings were inconclusive and should be cautiously interpreted and suggested well-designed animal and human studies of longer follow-up periods are required before implementing such a technique in regular clinical practice.

\section{Buccal bone wall: Considerations for clinical management}

After implant placement by application of the OD technique in an area with insufficient bone width, a thin buccal bone wall is created which could be considered as comparable to this following immediate implant placement. In this case, buccal bone resorption and soft-tissue recession both related to a thin buccal bone wall could also occur. Buccal bone width is an important factor in implant surgery planning and outcomes, because buccal bone resorption can reduce soft tissue stability and thus lead to implant exposure and lead to an unfavorable esthetic result. A minimum buccal bone wall width of $2 \mathrm{~mm}$ at the implant site has been recommended to ensure maintenance of the crest around an implant. It would be prudent to have a greater minimal buccal bone width criterion for immediate implant placement to account for post-extraction dimensional changes $[55,56]$. However, such a criterion is met in only a minority of anterior maxilla sites, where buccal wall widths $\leq 1 \mathrm{~mm}$ are commonly found. Consequently, in most clinical situations, augmentation is needed to achieve adequate bony contours around implants [56].

Connective tissue grafts (CTGs), or more recently developed collagen-based matrices (CMs), can be applied prior to or during an implant placement procedure to increase soft-tissue thickness (STTh) [57-59]. Notably, peri-implant soft-tissue augmentation with a novel tridimensional acellular dermal CM, composed of natural types I and III collagen without any artificial crosslinking. The histological results 6 to 12 months after soft tissue augmentation showed that this biomaterial has been completely replaced by health connective tissue and yield an STTh increase between $72 \%$ and $80 \%$ [58,60]. Although insufficient STTh does not compromise the mechanical stability of implants per se, it is associated with peri-implant soft-tissue recession and subsequent bone wall resorption [57-59]. In a systematic review, ViñaAlmunia et al. [61] reported that mucogingival surgery-based ridge preservation techniques can minimize buccal bone crest resorption effectively and that biomaterials, such as CMs, could be as effective as autologous CTGs.

Buccal site augmentation with a CTG $[57,59]$ or CM $[58,61]$ to prevent buccal bone resorption and soft tissue recession may also be helpful and/or indicated in areas of implant placement by use of OD osteotomy. However, it was not described or discussed in any of the available literature.

\section{Discussion}

OD was introduced as a novel approach in implant surgery promising to increase biomechanical PIS, bone mineral density, and bone-to-implant contact $[15,16,19,46]$. The OD-burs enable bone condensation through compaction as well as the increase of bone density, and PIS [33]. This osteotomy approach has been shown to be applicable in the context of low-bone density, which 
becomes increasingly common with advancing age, and to shorten recovery owing to full $\mathrm{OI}$ within as little as 14 weeks in human patients $[15,16,46]$. Additionally, BIC levels were greater in both CW-OD and CCW-OD than in SD, though changes in BIC were negligible over time $[9,24,26,33,43]$. Trisi et al. [33] demonstrated that implants inserted using OD osteotomy showed statistically higher biomechanical values of $30 \%$ to $40 \%$ than implants inserted with conventional drills. Alifarag et al. [24] provided a detailed overview of the history of endosteal implants and their ability to facilitate healing following orthopedic and dental treatments. They emphasized that endosteal implant success depends on PIS, which is sensitive to bone quality and quantity. In this context, OD leads to improved PIS relative to SD, leading to better outcomes $[9,33]$. With regard to the in vivo sheep hip model employed in the presently reviewed studies is appropriate for preclinical dental implant studies owing to its low-density structure, approximating that found in the jaws of elderly human patients $[9,24,26,33,43,44]$.

Moreover, performing experiments in living mammals facilitates clinical translation to humans. Employing eloquent histological preparations and statistical analyses, studies have provided clear demonstrations that OD increases torque values, which are indicative of better PIS [24,26,9,43,33,44]. More specifically, the study by Alifarag et al. [24] demonstrated that OD was associated with greater insertion force than SD despite the fact that osteotomies made by SD are smaller than those made by OD. Although PIS was achieved with both OD and SD, it required 3 weeks to achieve following SD due to bone loss during drilling. [24] Notably, the study by Lahens et al. [26] wherein 72 osteotomies in sheep were subjected to histomorphic analysis, OD resulted in better PIS and OI of implants in low-density bone than was achieved with SD. Lahens et al. study further confirmed the superior performance of OD over conventional subtractive implantation methods [9]. Consistent with the Lopez et al. [43], Slete et al. showed that OD led to BIC superior that that achieved with SD or SO [13].

The case reported by de Mello Machado et al. [48] was similar to Huwais [15]; Hofbauer \& Huwais [46] in that the subjects of the studies were males, but distinct in that the patient being only 44 years old was substantially younger than the patients described by Huwais [15]; Hofbauer \& Huwais [46] who were in their 60s. Additionally, de Mello Machado et al. [48] patient was being treated for an intractable root fracture rather than for a missing tooth with advanced alveolar ridge resorption, and therefore underwent extraction with immediate implantation. All three case reports emphasized the rapidity of healing that was achieved with OD relative to their experiences with SD and/ or SO. The review by Tretto et al. [52] is unique in that it focused on the tools used rather than the overall procedure. They did not find significant differences in the survival of implants among conventional drills (CDs), PDs and OTs. However, they noted a particularly weak inflammatory response in osteotomies created with PDs. Moreover, the use of OD burs showed promising results to be applied in clinical research; however, were evaluated only in animal studies in short periods. From the general observation of this publication, OT did not improve the bone-implant interface in comparison with CDs, but it is worth mentioning that OTs have other uses, such as to perform bone expansion [52].

Also, it was found that PDs seemed to provide better biologic response when compared to CDs. Er: YAG Laser was shown not to provide relevant benefits. ODs showed promising and encouraging results because of the significant increase in the biomechanical properties. Additionally, this study validated the bone expansion attitude of OD showing that wider implant diameter could be inserted in narrow ridge without creating bone dehiscence or fenestration [52]. Unlike conventional bone-excavating osteotomy, OD compacts and autografts particulate bone, yielding a boneretaining osteotomy $[44,45]$. OD also yields higher insertion torque than SD, which enhances PIS. Relative to CW-OD, CCWOD has produced better results with a reduced risk of fraction, though both approaches yield faster healing than SD. CCW-OD also results in better BIC and bone value percentages than traditional techniques $[5,9,16,18,31,48]$.

\section{Conclusion}

Although the actual OD-findings are encouraging and many clinicians are very confident about the success of this method, it would be premature to make conclusions regarding long-term clinical outcomes. It should be noted that the obtained results are either based on case reports or on clinical studies with a relatively small number of patients and short observation period or on animal studies or on in vitro experiments. Limited data exist on the tissue response in compromised periodont, or healing time as well as on the use of different implant geometries. Furthermore, no data exist regarding possible atrophy of the created thin buccal bone wall and its protection by application of OD preparation combined with a CTG. Long-term controlled clinical studies are necessary to define the limitations and to elucidate the clinical utility of the OD osteotomy before implementing such technique in regular daily implant surgery.

\section{References}

1. Zafiropoulos GG, Deli G, Bartee BK, Hoffmann O (2010) Single tooth implant placement and loading in fresh and regenerated extraction sockets. 5-year results. A case series using two different implant designs. J Periodontol 81(4): 604-615.

2. Hoffmann O, Bartee BK, Beaumont Chr, Kasaj A, Deli G, et al. (2008) Alveolar bone preservation in extraction sockets using non-resorbable high-density polytetrafluoroethylene (dPTFE) membranes. A retrospective non-randomized study. J Periodontol 79(8): 1355-1369.

3. Maló P, de Araújo Nobre M, Lopes A, Ferro A, Nunes M (2019) The Allon-4 concept for full-arch rehabilitation of the edentulous maxillae: A longitudinal study with 5-13 years of follow-up. Clin Implant Dent Relat Res 21(4): 538-549. 


\section{Advances in Dentistry \& Oral Health}

4. Tanaka K, Sailer I, Iwama R, Yamauchi K, Nogami S, et al. (2018) Relationship between cortical bone thickness and implant stability at the time of surgery and secondary stability after osseointegration measured using resonance frequency analysis. J Periodontal Implant Sci 48(6): 360-372.

5. Dayan C, Geckili O, Bural C (2019) The influence of implant shape on primary stability of implants with a thread cutting and forming design: an ex vivo study. J Oral Implantol 45(3):181-185.

6. Branemark PI (1983) Osseointegration and its experimental background. J Prosthet Dent 50(3): 399-410.

7. Brånemark PI, Adell R, Albrektsson T, Lekholm U, Lundkvist S, et al. (1983) Osseointegrated titanium fixtures in the treatment of edentulousness. Biomaterials 4(1): 25-28.

8. Albrektsson T, Brånemark PI, Hansson HA, Lindström J (1981) Osseointegrated titanium implants. Requirements for ensuring a longlasting, direct bone-to-implant anchorage in man. Acta Orthop Scand 52(2): $155-170$

9. Lahens B, Neiva R, Tovar N, Alifarag AM, Jimbo R, et al. (2016) Biomechanical and histologic basis of osseodensification drilling for endosteal implant placement in low density bone. An experimental study in sheep. J Mech Behav Biomed Mater 63: 56-65.

10. Gomes JB, Campos FE, Marin C, Teixeira HS, Bonfante EA, et al. (2013) Implant biomechanical stability variation at early implantation times in vivo: an experimental study in dogs. Int J Oral Maxillofac Implant 28(3): e128-e134.

11. Albrektsson T, Wennerberg A (2004) Oral implant surfaces: Part 1 Review focusing on topographic and chemical properties of different surfaces and in vivo responses to them. Int J Prosthodont 17(5): 536543

12. Albrektsson T, Wennerberg A (2004) Oral implant surfaces: Part 2 - Review focusing on clinical knowledge of different surfaces. Int Prosthodont 17(5): 544-564.

13. Slete FB, Olin P, Prasad H (2018) Histomorphometric comparison of 3 osteotomy techniques. Implant Dent 27(4): 424-428.

14. Büchter A, Kleinheinz J, Wiesmann HP, Kersken J, Nienkemper M, et al. (2005) Biological and biomechanical evaluation of bone remodelling and implant stability after using an osteotome technique. Clin Ora Implants Res 16(1): 1-8.

15. Huwais S (2015) Enhancing implant stability with osseoidensification a case report with two years follow up. J Impl Pr 8(X): 28-34.

16. Huwais S, Meyer E (2017) A novel osseous densification approach in implant osteotomy preparation to increase biomechanical primary stability, bone mineral density, and bone-to-implant contact. Int J Oral Maxillofac Implants 32(1): 27-36

17. Greenstein G, Cavallaro J (2017) Implant insertion torque: its role in achieving primary stability of restorable dental implants. Compend Contin Educ Dent 38(2): 88-95.

18. Wang L, Wu Y, Perez KC, Hyman S, Brunski JB, et al. (2017) Effects of condensation on peri-implant bone density and remodeling. J Dent Res 96(4): 413-420

19. Huwais S (2014) Autografting osteotome. Geneva, Switzerland: World Intellectual Property Organization. Publication.

20. Jimbo R, Tovar N, Marin C, Teixeira HS, Anchieta RB, et al. (2014) The impact of a modified cutting flute implant design on osseointegration. Int J Oral Maxillofac Surg 43(7): 883-888.

21. Summers RB (1994) A new concept in maxillary implant surgery: the osteotome technique. Compendium 15(2):152-160.
22. Summers RB (1994) The osteotome technique: Part 2 - The ridge expansion osteotomy (REO) procedure. Compendium 15(4): 422-434.

23. Summers RB (1994) The osteotome technique: Part 3 - Less invasive methods of elevating the sinus floor. Compend Contin Educ Dent 15(6): 698.

24. Alifarag AM, Lopez CD, Neiva RF, Tovar N, Witek L, et al. (2018) Atemporal osseointegration: Early biomechanical stability through osseodensification. J Orthop Res 36(9): 2516-2523.

25. Pai U, Rodrigues S, Talreja K, Mundathaje M (2018) Osseodensification - A novel approach in implant dentistry. J Indian Prosthodont Soc 18(3): 196-200

26. Lahens B, Lopez CD, Neiva RF, Bowers MM, Jimbo R, et al. (2019) The effect of osseodensification drilling for endosteal implants with different surface treatments: A study in sheep. J Biomed Mater Res Part B Appl Biomater 107(3): 615-623.

27. Turkyilmaz I, McGlumphy EA (2008) Influence of bone density on implant stability parameters and implant success: a retrospective clinical study. BMC Oral Health 8: 32.

28. Beer A, Gahleitner A, Holm A, Tschabitscher M, Homolka P (2003) Correlation of insertion torques with bone mineral density from dental quantitative CT in the mandible. Clin Oral Implants Res 14(5): 616-620.

29. Abrahamsson I, Linder E, Lang NP (2009) Implant stability in relation to osseointegration: An experimental study in the Labrador dog. Clin Oral Implants Res 20(3): 313-318.

30. Chuang SK, Wei LJ, Douglass CW, Dodson TB (2002) Risk factors for dental implant failure: A strategy for the analysis of clustered failuretime observations. J Dent Res 81(8): 572-577.

31. Padmanabhan TV, Gupta R (2010) Comparison of crestal bone loss and implant stability among the implants placed with conventional procedure and using osteotome technique: a clinical study. J Oral Implantol. 36(6): 475-483.

32. Meredith N (1998) Assessment of implant stability as a prognostic determinant. Int J Prosthodont. 11(5): 491-501.

33. Trisi P, Berardini M, Falco A, Podaliri Vulpiani M (2016) New osseodensification implant site preparation method to increase bone density in low-density bone: In vivo evaluation in sheep. Implant Dent. 25(1): 24-31.

34. Yoon HG, Heo SJ, Koak JY, Kim SK, Lee SY (2011) Effect of bone quality and implant surgical technique on implant stability quotient (ISQ) value. J Adv Prosthodont 3(1): 10-15.

35. Alghamdi H, Anand PS, Anil S (2011) Undersized implant site preparation to enhance primary implant stability in poor bone density: a prospective clinical study. Oral Maxillofac Surg 69(12): e506-512.

36. Al-Marshood MM, Junker R, Al-Rasheed A, Al Farraj Aldosari A, Jansen JA, et al. (2011) Study of the osseointegration of dental implants placed with an adapted surgical technique. Clin Oral Implants Res 22(7): 753759.

37. Fanuscu MI, Chang TL, Akça K (2007) Effect of surgical techniques on primary implant stability and peri-implant bone. J Oral Maxillofac Surg 65(12): 2487-2491.

38. Al-Marshood MM, Junker R, Al-Rasheed A, Al Farraj Aldosari A, Jansen JA, et al. (2011) Study of the osseointegration of dental implants placed with an adapted surgical technique. Clin Oral Implants Res 22(7): 753759.

39. Turkyilmaz I, Tozum TF, Tumer C, Ozbek EN (2006) Assessment of correlation between computerized tomography values of the bone, and maximum torque and resonance frequency values at dental implant placement. J Oral Rehabil 33(12): 881-888. 


\section{Advances in Dentistry \& Oral Health}

40. Turkyilmaz I, Tozum TF (2019) Enhancing primary implant stability by undersizing implant site preparation: A human cadaver study. J Stomatol Oral Maxillofac Surg S2468-7855.

41. Dhore CR, Snel SJ, Jacques SVN, Naert IE, Walboomers XF, et al. (2008) In vitro osteogenic potential of bone debris resulting from placement of titanium screw-type implants. Clin Oral Implants Res 19(6): 606-611.

42. Donath K, Breuner G (1982) A method for the study of undecalcified bones and teeth with attached soft tissues: The säge-schliff (sawing and grinding) technique. J Oral Pathol Med 11(4): 318-326.

43. Lopez CD, Alifarag AM, Torroni A, Tovar N, Diaz-Siso JR, et al. (2017) Osseodensification for enhancement of spinal surgical hardware fixation. J Mech Behav Biomed Mater 69: 275-281.

44. Oliveira PGFP, Bergamo ETP, Neiva R, Bonfante EA, Witek L, et al. (2018) Osseodensification outperforms conventional implant subtractive instrumentation: A study in sheep. Mater Sci Eng C 90: 300-307.

45. Tian JH, Neiva R, Coelho PG, Witek L, Tovar NM, et al. (2019) Alveolar ridge expansion: Comparison of osseodensification and conventional osteotome techniques. J Craniofac Surg 30(2): 607-610.

46. Hofbauer A, Huwais S (2016) Osseondensification facilitates ridge expansion with enhanced implant in the maxilla. Part II. J Impl Pr 8: $18-25$.

47. Koutouzis T, Huwais S, Hasan F, Trahan W, Waldrop T, et al. (2019) Alveolar ridge expansion by osseodensification-mediated plastic deformation and compaction autografting: a multicenter retrospective study. Implant Dent 28(4): 349-355.

48. De Mello Machado RC, da Gama CS, Batista SH, Rizzo D, Valiense H, et al. (2018) Tomographic and clinical findings, pre-, trans-, and postoperative, of osseodensification in immediate loading. Int J Growth Factors Stem Cells Dent 1(3): 101-105.

49. Da Rosa JCM, Pértile de Oliveira Rosa AC, Huwais S (2019) Use of the immediate dentoalveolar restoration technique combined with osseodensification in periodontally compromised extraction sites. Int J Periodontics Restorative Dent 39(4): 527-534.

50. Witek L, Neiva R, Alifarag A, Shahraki F, Sayah G, et al. (2019) Absence of healing impairment in osteotomies prepared via osseodensification drilling. Int J Periodontics Restorative Dent 39(1): 65-71.

51. Almutairi AS, Walid MA, Alkhodary MA (2018) The effect of osseodensification and different thread designs on the dental implant primary stability. F1000Res 7: 1898.
52. Tretto PHW, Fabris V, Cericato GO, Sarkis-Onofre R, Bacchi A (2019) Does the instrument used for the implant site preparation influence the bone-implant interface? A systematic review of clinical and animal studies. Int J Oral Maxillofac Surg 48(1): 97-107.

53. El-Kholey KE, Elkomy A (2019) Does the drilling technique for implant site preparation enhance implant success in low density bone? A systematic review. Implant Dent 28(5): 500-509.

54. Elsayyad AA, Osman RB (2019) Osseodensification in implant dentistry: A critical review of the literature. Implant Dent 28(3): 306-312.

55. Merheb J, Vercruyssen M, Coucke W, Beckers L, Teughels W, et al. (2017) The fate of buccal bone around dental implants. A 12-month postloading follow-up study. Clin Oral Implants Res 28(1): 103-108.

56. Huynh-Ba G, Pjetursson BE, Sanz M, Cecchinato D, Ferrus J, et al. (2010) Analysis of the socket bone wall dimensions in the upper maxilla in relation to immediate implant placement. Clin Oral Impl Res 21(1): 3742.

57. Kan JY, Rungcharassaeng K, Lozada JL (2005) Bilaminar subepithelial connective tissue grafts for immediate implant placement and provisionalization in the esthetic zone. J Calif Dent Assoc 33(1): 865871.

58. Zafiropoulos GG, John G (2017) Use of collagen matrix for augmentation of the peri-implant soft tissue at the time of immediate implant placement. J Contemp Dent Pract 18(5): 386-391.

59. Kan JY, Rungcharassaeng K, Lozada JL, Zimmerman G (2011) Facial gingival tissue stability following immediate placement and provisionalization of maxillary anterior single implants: a 2- to 8-year follow-up. Int J Oral Maxillofac Implants 26(1): 179-187.

60. Papi P, Pompa G (2018) The use of a novel porcine derived acellular dermal matrix (Mucoderm) in peri-implant soft tissue augmentation: preliminary results of a prospective pilot cohort study. Biomed Res Int 2018: 6406051

61. Viña-Almunia J, Candel-Martí ME, Cervera-Ballester J, García-Mira B, Calvo-Guirado JL, et al. (2013) Buccal bone crest dynamics after immediate implant placement and ridge preservation techniques: review of morphometric studies in animals. Implant Dent 22(2): 155160.

\begin{tabular}{l} 
Your next submission with Juniper Publishers \\
will reach you the below assets \\
- Quality Editorial service \\
- Swift Peer Review \\
- Reprints availability \\
- E-prints Service \\
- Manuscript Podcast for convenient understanding \\
- Global attainment for your research \\
- Manuscript accessibility in different formats \\
( Pdf, E-pub, Full Text, Audio) \\
- Unceasing customer service \\
Track the below URL for one-step submission \\
https://juniperpublishers.com/online-submission.php \\
\hline
\end{tabular}

